\section{RESISTIVE INTERCHANGE INSTABILITY CRITERION IN \\ ANISOTROPIC TOROIDAL SYSTEMS}

J.L. Johnson

Plasma Physics Laboratory, Princeton Univers. $v$

P.0. Box 451

Princeton, New Jersey 08544

and

R.J. Hastie

Culham Laboratory, U.K.A.E.A.

Abingdon, Oxfordshire

England

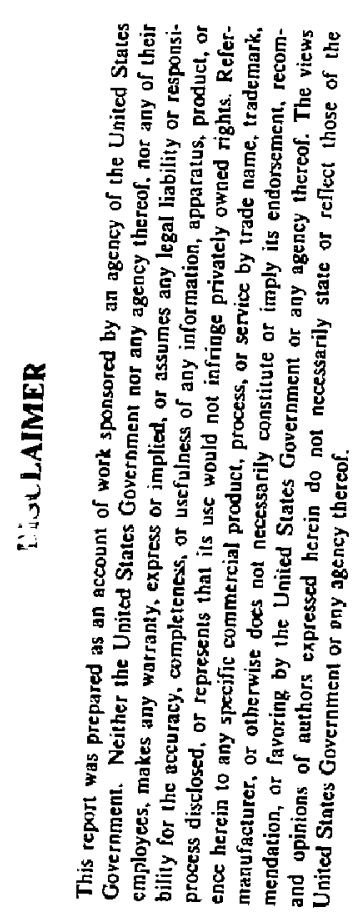

\title{
ABSTRACT
}

Ideal (Mercier) and resistive criteria for localized magnetohydrodynamic instabilities are derived for general toroidal systems with anisotropic pressure.

\section{MASTER}


The ideal (Mercier) and resistive criteria for localized modes have been useful for predicting stability behavior of stellarators and tokamaks with scalar pressure. ${ }^{1}$ Since they can be evaluated easily on each magnetic surface, these criteria are often included as part of a numerical equilibrium calculation. They provide a measure of the energy that is available locally to drive an instability and thus can even provide insight into the nonlinear evolution of the modes. Indeed, it has recently been shown ${ }^{2}$ that the resistive criterion governs the nonlinear evolution of magnetic islands. Our purpose here is to generalize these criteria and not to evaluate the wave properties of the magnetohydrodynamic modes associated with them. The ideal criterion has been generalized to include anisotropic pressure in axisymmetric systems. ${ }^{3}$ Here, we derive this and the resistive criterion for arbitrary toroidal systems. Since the plasma anisotropy created by auxiliary heating in the present and future generations of tokamaks and stellarators can modify the equilibrium properties of the devices, this generalization seems useful.

In the Appendix we follow the derivation of a set of averaged equations that was given by Johnson and Greene. 4 Since these equations are similar to those considered by Glasser et al. 1 we can then identify with that work to obtain the criteria:

Ideal Interchange,

$$
D_{I} \equiv E+H-\frac{1}{4}<0 \quad \text { for stability; }
$$

$$
\begin{aligned}
& \text { Resistive Interchange, } \\
& \mathrm{D}_{\mathrm{R}} \equiv \mathrm{E}+\mathrm{H}^{2}=\mathrm{D}_{\mathrm{I}}+\left(\mathrm{H}-\frac{1}{2}\right)^{2}<0 \text { for stability. }
\end{aligned}
$$


Here

$$
\begin{aligned}
& E \equiv \frac{\left\langle B^{2} / \sigma|\nabla V|^{2}\right\rangle}{n^{2}}\left[\left\langle\frac{\nabla V \cdot \nabla\left(p_{\perp}+B^{2} / 2\right)}{\tau \sigma B^{2}|\nabla V|^{2}}\left(\left.\sigma \frac{\partial p_{1}}{\partial V}\right|_{B}+\left.\tau \frac{\partial P_{\|}}{\partial V}\right|_{B}\right)\right\rangle\right. \\
& \left.+\left\langle\frac{\sigma(J \cdot B)^{2}}{B^{2}|\nabla V|^{2}}\right\rangle-\frac{\left(\left\langle\mathrm{J} \cdot \mathrm{B} /|\nabla v|^{2}\right\rangle\right)^{2}}{\left\langle B^{2} / \sigma|\nabla V|^{2}\right\rangle}+\phi v^{\prime}\left\langle\frac{\sigma \mathrm{J} \cdot \mathrm{B}}{B^{2}} \frac{\partial}{\partial \zeta} \frac{\nabla v \cdot \nabla u}{|\nabla v|^{2}}\right\rangle\right], \\
& \left.H \equiv \frac{\left\langle B^{2} / a|\nabla V|^{2}\right\rangle}{A}\left[\frac{\left\langle J \cdot B /|\nabla V|^{2}\right\rangle}{\left\langle B^{2} / \sigma|\nabla V|^{2}\right\rangle}\right]-\frac{\langle j \cdot \theta\rangle}{\left\langle B^{2} / \sigma\right\rangle}\right], \\
& n \equiv \psi^{\prime} x^{\prime \prime}-x^{\prime} \psi^{\prime \prime}, \\
& \phi \psi^{\prime} \frac{\partial}{\partial \zeta} \frac{\nabla V \cdot \nabla u}{|\nabla V|^{2}}=\Lambda-\frac{B \times \nabla V \cdot[\nabla \times(\underset{\sim}{B} \times \nabla V)]}{|\nabla V|^{4}} \text {, } \\
& \langle A\rangle \equiv \frac{\oint A d \ell / B}{\oint d \ell / B}, \quad 0 \equiv 1-\left.\frac{\partial P_{1}}{B \partial B}\right|_{V}, \quad \tau \equiv 1+\left.\frac{\partial P_{1}}{B \partial B}\right|_{V} .
\end{aligned}
$$

The parameter A measures the local shear, with primes representing derivatives with respect to $V$.

Since all of the functions that enter these relations are determined in an equilibrium calculation, these expressions can be evaluated easily. A $(V, \theta, \zeta)$ set of Hamada coordinates with a unit Jacobian was used for the calculation, with $V$ the volume inside the relevant magnetic surface. Use of the function $u=m \theta-n \zeta$, such that $\underline{B}=\nabla V \times \nabla u$ on the surface of interest rather than $\theta$, simplifies the work.

In the isotropic limit, the pressure functions, $P_{\perp}(V, B)$ and $P_{\|}(V, B)$, 
become surface functions and the criteria reduce to their well-known scalar pressure forms. 1 The first term in $E$ is clearly associated with the normal component of the magnetic field line curvature. The remaining terms in $E$ and $H$ can be identified with the geodesic curvature through its relation to the surface variation of the parallel current. As before, the major difference between the ideal and resistive criteria is the factor of $1 / 4$ introduced into the ideal form by the shear. The modification associated with the different way that $H$ enters is due to the identification of $\nabla^{2} \underline{v}$ with $\xi$ rather than wb with $\xi$ in Ohm's law, resulting in a different weighting over the magnetic field lines.

\section{ACKNOWLEDGMENT}

Part of this work was supported by the U.S. Department of Energy Contract No. DE-ACO2-76-CHO3O73 with Princeton University. 
APPENDIX

We consider a toroidal equilibrium with anisotropic pressure such that

$$
\begin{aligned}
& J \times \underline{B}=\nabla \cdot \stackrel{P}{=}, \\
& P=p_{\perp} \|+\left(p_{1}-p_{\perp}\right) \frac{B B}{B^{2}} .
\end{aligned}
$$

It is convenient to define 5

$$
\begin{aligned}
& \underline{K} \equiv \nabla \times \sigma \underline{\sigma B}, \\
& \sigma \equiv i+\frac{\left(p_{\perp}-P_{\|}\right)}{B^{2}}, \\
& T \equiv 1+\left.\frac{\partial P_{\perp}}{B \partial B}\right|_{V} .
\end{aligned}
$$

Then

$$
\begin{aligned}
& \underline{K} \times \underline{B}=\left.\frac{\partial p_{\|}}{\partial V}\right|_{B} \nabla V, \\
& \left.\frac{\partial p_{V}}{B \partial B}\right|_{V}=1-\sigma .
\end{aligned}
$$

The $\underline{B}$ and $\underline{K}$ lines lie on magnetí surfaces, so that much of the formalism that has been developed for isotropic systems can be applied. The plasma current is given by 


$$
\begin{aligned}
& I=\frac{B^{2}}{|\nabla V|^{2}} \frac{(\sigma-\tau)}{\left(\sigma a p_{\perp} /\left.\partial V\right|_{B}+\tau \partial p_{\eta} /\left.\partial V\right|_{B}\right)} \underline{B} \cdot \nabla\left(\frac{\sigma J \cdot B}{B^{2}}\right) \nabla V \\
& +\left(\frac{\nabla V \cdot \nabla p_{1}}{\sigma B^{2}|\nabla V|^{2}}+\frac{(\sigma-1) \nabla V \cdot \nabla 8^{2}}{2 \sigma B^{2}|\nabla V|^{2}}\right) \underset{\sim}{B} \times \nabla V+\left(\frac{\underset{B}{J}-\frac{B}{B^{2}}}{B} \underset{\sim}{B}\right.
\end{aligned}
$$

with

$$
\underline{B} \cdot \nabla\left(\frac{\sigma J \cdot g}{B^{2}}\right)=\frac{B \times \nabla v \cdot \nabla B^{2}}{2 \sigma B^{4}}\left(\left.\sigma \frac{\partial p_{1}}{\partial V}\right|_{B}+\left.\tau \frac{\partial p_{y}}{\partial V}\right|_{B}\right) .
$$

He adopt a Hamada $V, \theta, \tau$ coordinate system with unit Jacobian. $V$ is the volume inside a magnetic surface, and $\theta$ and $\zeta$ are angular coordinates that increase by unity in going once around the torus the short and long ways, respectively. We write

$$
\underline{B}=\nabla V \times\left[w^{\prime}(V) \nabla \theta-x^{\prime}(V) \nabla \zeta\right]
$$

with $W^{\prime}(v)$ and $x(V)$ the magnetic fiuxes the long and short ways. Primes denote derivatives with respect to $V$. Since we are concerned with modes localized near a surface $v_{0}$, on which the safety factor $\psi^{\prime}\left(v_{0}\right) / x^{\prime}\left(v_{0}\right)=m / n$ is rational, we transform to an $x, u, z$ coordinate system where

$$
\begin{aligned}
& x \equiv v-v_{0}, \\
& u \equiv m \theta-n \varphi,
\end{aligned}
$$

and write 


$$
\begin{aligned}
& \Lambda \equiv \psi^{\prime}\left(v_{0}\right) x^{+1}\left(v_{0}\right)-x^{\prime}\left(v_{0}\right) \psi^{\prime}+\left(v_{0}\right), \\
& \phi \equiv \psi^{\prime}\left(v_{0}\right) / m=x^{\prime}\left(v_{0}\right) / n .
\end{aligned}
$$

Then

$\underline{B}=\nabla V \times \nabla u\left[1+\psi^{\prime}\left(V_{0}\right) / \psi^{\prime}\left(V_{0}\right)\right]+\nabla V \times \nabla \zeta \frac{\Delta x}{\psi^{\prime}}+\cdots$.

We use the usual linearized MHD equations for the stability analysis,

$$
\begin{aligned}
& a q^{2} \xi=\underline{j} \times \underline{B}+\underline{J} \times \underline{b}-\nabla \cdot \hat{p}, \\
& \underline{b}-\frac{\vec{p}}{q} \nabla^{2} \underline{b}=\nabla \times(\underline{\xi} \times \underline{B}),
\end{aligned}
$$

with the modes varying with time as $\exp (a t)$ and the resistivity $n$ a constant. Lower-case symbols, $\xi, j, b, \hat{p}_{\perp}$, and $\hat{p}_{\|}$denote perturbed quantities.

The determination of the quantities $D_{I}$ and $D_{R}$ in the final averaged equations is not affected by the choice of the equations of state for $p_{\|}$and $p_{\perp}$. For simplicity we adopt a model in which the distribution functions are merely convected with the field lines. Then,

$$
\begin{aligned}
& \hat{p}_{\|}=(1-\sigma) \frac{\underline{b} \cdot \underline{B}}{B^{2}}-\left.\frac{\partial P_{\|}}{\partial V}\right|_{B} \frac{\xi \cdot \nabla V}{|\nabla V|^{2}}, \\
& \hat{p}_{\perp}=\left.(\tau-1) \frac{\frac{b \cdot B}{B^{2}}}{\partial V}\right|_{B} \frac{\partial p_{\perp}}{|\nabla V|^{2}} .
\end{aligned}
$$


This is the appropriate generalization to anisotropic equilibria of the pressure convection model of scalar pressure MHD (adiabatic index $\gamma \equiv 0$ ) in which no sound waves propagate. As noted previously 6,7 this permits new instabilities. However, it does not prevent us from identifying the anisotropic $D_{R}$ and $D_{I}$.

It is convenient to decompose the perturbations as

$$
\begin{aligned}
& \underline{\xi}=\xi_{V} \frac{\nabla V}{|\nabla V|^{2}}+\xi_{s} \frac{\frac{B \times \nabla V}{B^{2}}+\xi_{b} \frac{B}{B^{2}},}{\underline{b}}=b_{V} \frac{\nabla V}{|\nabla V|^{2}}+b_{s} \frac{B \times \nabla V}{B^{2}}+b_{B} \frac{B}{B^{2}},
\end{aligned}
$$

and to introduce the orderings

$$
\begin{aligned}
& n-\varepsilon^{8}, q-\varepsilon^{2}, \frac{\partial}{\partial x}-\varepsilon^{-3}, \frac{\partial}{\partial L}-\varepsilon^{-1}, \frac{\partial}{\partial \zeta}-\varepsilon^{0}, \\
& \xi_{S}=\varepsilon^{0}, \xi_{V}-b_{S}-b_{B}-\varepsilon^{2}, b_{V}-\varepsilon^{4} .
\end{aligned}
$$

This rather artificial choice of ordering was illtroduced in Ref. 4 because it simplified the algebra. Similar results follow from the usual a/ax $n^{1 / 3}$ ordering. 1 The calculation can be made in the same way as in Appendix $B$ of Ref. 4. In lowest order, the condition that the perturbed field be divergence-free requires that

$$
\frac{\partial b_{v}^{(4)}}{\partial x}+\frac{1}{d} \frac{\partial b_{s}^{(2)}}{\partial u}=0 .
$$

The VV component of the induction equation, Eq. (A10), leads to 


$$
\begin{aligned}
& \psi^{\prime} \frac{\partial \varepsilon_{V}{ }^{(2)}}{\partial \zeta}=0, \\
& \psi^{\prime} \frac{\partial \varepsilon_{V}(3)}{\partial \zeta}=0, \\
& b_{V}{ }^{(4)}-\frac{\eta}{q}|\nabla V|^{2} \frac{a^{2} b_{V}{ }^{(4)}}{\partial x^{2}}=\psi^{\prime} \frac{\partial \varepsilon_{V}}{\partial \zeta}+\frac{\Delta x}{\phi} \frac{\partial \varepsilon_{V}}{\partial u} .
\end{aligned}
$$

The component of this equation along $B$ gives

$$
\begin{aligned}
& \frac{\partial \xi_{V}(2)}{\partial x}+\frac{1}{\partial} \frac{\partial \xi_{S}(0)}{\partial u}=0, \\
& \frac{\partial \xi_{V}(3)}{\partial x}+\frac{1}{0} \frac{\partial \xi_{S}(1)}{\partial u}+\frac{B \times \nabla V \cdot \nabla \zeta}{B^{2}} \frac{\partial \xi_{S}(0)}{\partial \zeta}=0, \\
& \frac{\partial \xi_{V}(4)}{\partial x}+\frac{\nabla V \cdot \nabla u}{|\nabla V|^{2}} \frac{\partial \xi_{V}(2)}{\partial u}+\frac{1}{\phi} \frac{\partial \xi_{S}(2)}{\partial u}+\frac{B \times \nabla V \cdot \nabla \zeta}{B^{2}} \frac{\partial \xi_{S}(1)}{\partial \zeta}=0 .
\end{aligned}
$$

The $\mathrm{B} \times$ oV component of the induction equation is not needed because of the relation imposed by the $\nabla \cdot \underline{b}=0$ constraint. In Ref. 4, we used the condition that $\hat{p}-\varepsilon^{2}$ to make $\nabla \cdot \xi-\varepsilon^{2}$. We thus tied $\partial \xi_{B} / a \xi_{0}$ to $\xi_{\perp}$ and kept $\nabla \cdot g^{(2)}$ as an independent variable. Here, our incompressibility assumption in the equation of state removes tinis constraint. For this reason we only have to go one order lower in this equation than before.

We now turn to the momentum equation. The $\nabla V$ component requires that

$$
\frac{\partial}{\partial x}\left(\hat{p}_{1}^{(2)}+b_{B}^{(2)}\right)=0,
$$




$$
\begin{aligned}
& \frac{\partial}{\partial x}\left(\hat{p}_{\perp}^{(3)}+b_{B}{ }^{(3)}\right)=0, \\
& \frac{\partial}{\partial x}\left(\hat{p}_{\perp}^{(4)}+b_{B}^{(4)}\right)+\frac{\nabla V \cdot \nabla u}{|\nabla V|^{2}} \frac{\partial}{\partial u}\left(\hat{p}_{\perp}^{(2)}+b_{B}^{(2)}\right)=0 .
\end{aligned}
$$

The $\mathrm{B} \times \nabla V$ component gives

$$
\begin{aligned}
& \frac{\partial}{\partial u}\left(\hat{p}_{\perp}^{(2)}+b_{B}^{(2)}\right)=0 \text {, } \\
& -\frac{B^{2}}{\phi} \frac{\partial}{\partial U}\left(\hat{p}_{\perp}^{(3)}+b_{B}^{(3)}\right)-\underline{B} \times \nabla V+\nabla \zeta \frac{\partial}{\partial \zeta}\left(\hat{p}_{\perp}^{(2)}+b_{B}^{(2)}\right) \\
& +B^{2} \psi \cdot \frac{\partial}{\partial c}\left(\frac{a|\nabla V|^{2}}{B^{2}} b_{s}^{(2)}\right)+\frac{\tau B^{2} v \cdot a / a c\left(o j \cdot B / B^{2}\right)}{\left[\sigma a p_{\perp} /\left.a V\right|_{B}+\tau \partial p_{1} /\left.\partial V\right|_{B}\right]}\left(2 b_{B}(2)+p_{\perp}(2)-p_{1}(2)\right)=0 \text {, } \\
& -\frac{B^{2}}{\phi} \frac{\partial}{\partial u}\left(\dot{p}_{1}^{(4)}+b_{B}{ }^{(4)}\right)-B \times \nabla V \cdot \nabla \zeta \frac{\partial}{\partial \tau}\left(\dot{p}_{1}^{(3)}+b_{B}{ }^{(3)}\right) \\
& +B^{2} \psi \cdot \frac{\partial}{\partial \zeta}\left(\frac{a|\nabla V|^{2}}{B^{2}} b_{S}^{(3)}\right)+\frac{\tau \theta^{2} \psi \cdot \partial / \partial \zeta\left(\sigma J \cdot B / B^{2}\right)}{\left.\left|\sigma \partial p_{\perp} / \partial V\right|_{B}+\tau \partial p_{1} /\left.\partial V\right|_{B}\right)}\left(2 b_{B}(3)+p_{\perp}^{(3)}-p_{\|}^{(3)}\right)=0 \text {. }
\end{aligned}
$$

It will turn out that, to the order we go, these two sets of equations contain the same information. Thus, it is expedient to use the usual annihilation operator, $\nabla \cdot \underset{g}{g} / B^{2} \times$, on the equation to get

$$
\begin{aligned}
& -\psi \cdot \frac{\partial}{\partial \tau}\left(\frac{\sigma|\nabla V|^{2}}{B^{2}} \frac{\partial b_{S}(2)}{\partial x}\right) \\
& +\frac{\left[\psi^{\prime} \partial / \partial \zeta\left(\sigma J \cdot B / B^{2}\right)\right]}{\left(\sigma \partial p_{\perp} /\left.\partial V\right|_{B}+\tau \partial p_{B} /\left.\partial V\right|_{B}\right]}\left[(\sigma-\tau) \frac{\partial b_{B}^{(2)}}{\partial x}+\sigma \frac{\partial \hat{p}_{\perp}^{(2)}}{\partial x}+\tau \frac{\partial \hat{p}_{\|}^{(2)}}{\partial x}\right]=0,
\end{aligned}
$$




$$
\begin{aligned}
& -v^{\prime} \frac{\partial}{\partial c}\left(\frac{a|\nabla v|^{2}}{B^{2}} \frac{a b_{s}(3)}{\partial x}\right) \\
& +\frac{\psi^{\prime} 3 / \partial 5\left(\sigma J-B_{-} / B^{2}\right)}{\left(\sigma \nabla p_{\perp} /\left.a V\right|_{B}+\tau \partial p_{\|} /\left.a V\right|_{B}\right.}\left[(\sigma-\tau) \frac{\partial b_{B}{ }^{(3)}}{\partial x}+\sigma \frac{\partial \hat{p}_{\perp}^{(3)}}{\partial x}+\tau \frac{\partial \hat{p}_{\|}(3)}{\partial x}\right]=0 \text {, } \\
& -\Delta q^{2} \frac{|\nabla V|^{2}}{B^{2}} \frac{\partial \xi_{s}(0)}{\partial x}=-\psi \frac{\partial}{\partial b}\left(\frac{\partial|\nabla V|^{2}}{B^{2}} \frac{\partial b_{s}(4)}{\partial x}+\frac{\sigma \nabla V \cdot \nabla u}{B^{2}} \frac{\partial b_{s}(2)}{\partial L}\right) \\
& -\frac{|\nabla V|^{2} \Lambda x}{\phi B^{2}} \frac{3^{2} b_{s}(2)}{\partial x \partial u} \\
& +\frac{\left[W^{\prime} \partial / \partial \zeta\left(\sigma J \cdot B / B^{2}\right)\right]}{\left(\sigma \partial p_{1} /\left.\partial V\right|_{B}+\tau \partial p_{\|} /\left.\partial V\right|_{B}\right)}\left\{\left[(\sigma-\tau) \frac{\partial b_{B}^{(4)}}{\partial x}+\sigma \frac{\partial \hat{p}_{L}^{(4)}}{\partial x}+\tau \frac{\partial \hat{p}_{\|}^{(4)}}{\partial x}\right]\right. \\
& \left.+\frac{\nabla V \cdot \nabla u}{|\nabla V|^{2}}\left\{(\sigma-\tau) \frac{\partial b_{B}(2)}{\partial u}+0 \frac{\partial \hat{p}_{1}^{(2)}}{\partial u}+\tau \frac{\partial \hat{p}_{\|}^{(2)}}{\partial u}\right]\right\} \\
& +\frac{\nabla V \cdot \nabla p_{1}}{\phi \sigma B^{2}|\nabla v|^{2}}\left(\frac{a b_{B}(2)}{\partial u}-\frac{\partial \hat{p}_{U}^{(2)}}{\partial u}\right) \\
& -\frac{\nabla V \cdot \nabla B^{2}}{2 \phi \sigma B^{2}|\nabla V|^{2}}\left[(\sigma-\tau) \frac{\partial b_{B}^{(2)}}{\partial u}+a \frac{\partial \hat{p}_{\perp}(2)}{\partial u}+\tau \frac{\partial \hat{p}_{\|}^{(2)}}{\partial u}\right] .
\end{aligned}
$$

The component along $B$ is not needed in the calculation, again because of the incompressibility constraint. 
It is convenient to decompose all of the variables into functions which are constant on a field line and those which vary along the line but with zero average value. Thus,

$$
\begin{aligned}
& \xi_{V}=\bar{\xi}_{V}(v, u)+\tilde{\xi}_{V}(x, u, \tau), \\
& \oint \tilde{\xi}_{V} \frac{d l}{B}=0,
\end{aligned}
$$

etc. Then we see that

$$
\tilde{\varepsilon}_{V}{ }^{(2)}=0
$$

from Eq. (A16) and, from Eq. (A17), that

$$
\begin{aligned}
& \tilde{\xi}_{S}^{(0)}=0, \\
& \frac{\partial \bar{\xi}_{S}(0)}{\partial u}=-\phi \frac{\partial \bar{\xi}_{V}(2)}{\partial x} .
\end{aligned}
$$

The perpendicular components of the momentum equation, Eqs. (A18) and (A19), give

$$
\hat{p}_{\perp}^{(2)}+b_{B}^{(2)}=0 \text {. }
$$

Combining this with the equations of state, Eqs. (A11) and (A12), gives

$$
\hat{p}_{\perp}^{(2)}=-b_{B}{ }^{(2)}=-\left.\frac{1}{\tau} \frac{\partial p_{1}}{\partial V}\right|_{B} \bar{\xi}_{V}(2) \text {, }
$$


$\tilde{p}_{\|}^{(2)}=\left(\left.\frac{(\sigma-\tau)}{\tau} \frac{\partial p_{1}}{\partial V}\right|_{B}-\left.\frac{\partial p_{\|}}{\partial V}\right|_{B}\right) \bar{\xi}_{V}^{(2)}$.

Inserting these and Eq. (A15) into Eq. (A20) and integrating with respect to 6, we obtain

$$
\begin{aligned}
& \frac{a^{2} b_{v}{ }^{(4)}}{\partial x^{2}}=\left[\frac{J \cdot B}{|\nabla V|^{2}}-\frac{B^{2} \oint J \cdot B /|\nabla V|^{2} d \ell / B}{\sigma|\nabla V|^{2} \oint B^{2} / \sigma|\nabla V|^{2} d \ell / B}\right] \frac{\frac{\partial}{\phi}}{\phi} \frac{\partial^{r} \bar{\xi}_{V}^{(2)}}{\partial x \partial u} \\
& +\frac{B^{2} \phi d \ell / B}{\sigma|\nabla V|^{2} \oint B^{2} / \sigma|\nabla V|^{2} \cdot d \ell / B} \frac{\partial^{2} \bar{\sigma}_{V}^{(4)}}{\partial x^{2}} .
\end{aligned}
$$

When we insert these into the higher order equations, we find that the resulting conditions on the next order independent variables are the same as those on the lowest order ones. Thus, we can set

$$
\xi_{v}^{(3)}=\xi_{s}^{(1)}=b_{v}^{(5)}=b_{s}^{(3)}=\hat{p}_{1}^{(3)}=\hat{p}_{1}^{(3)}=0 .
$$

We are now ready to treat the higher order equations. Averaging Eq. (A16) over 5 gives

$\bar{E}_{v}^{(4)} \oint \frac{\mathrm{d} \ell}{B}-\frac{\pi}{q} \oint|\nabla v|^{2} \frac{\partial^{2} b_{v}^{(4)}}{\partial x^{2}} \frac{d \ell}{B}=\frac{\Lambda x}{\phi} \oint \frac{d \ell}{B} \frac{\partial \bar{\xi}_{v}^{(2)}}{\partial u}$.

Insertion of $\mathrm{Eq}$. (A26) then provides a relation

$$
\begin{aligned}
& E_{V}{ }^{(4)}-\frac{n}{q} \frac{\oint B^{2} / \sigma d \ell / B}{\oint B^{2} / \sigma|\nabla V|^{2} d \ell / B} \frac{\partial^{2} B_{V}(4)}{\partial x^{2}}=\frac{\Delta x}{\phi} \frac{a \vec{F}_{y}^{(2)}}{\partial u}
\end{aligned}
$$

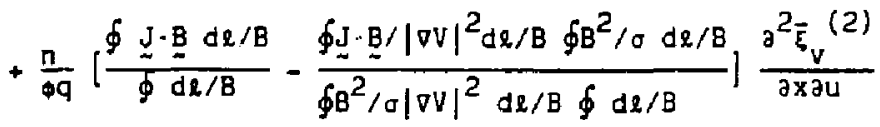


between $b_{V}(4)$ and $\xi_{V}(2)$. The perpendicular components of the momentum equation, together with the equations of state, give

$$
(a-\tau) b_{B}^{(4)}-\tau \hat{p}_{\|}^{(4)}-\sigma \hat{p}_{\perp}^{(4)}=\left(\left.a \frac{\partial p_{\perp}}{\partial V}\right|_{B}+\left.\tau \frac{\partial p_{\|}}{\partial V}\right|_{B}\right) \xi_{V}{ }^{(4)} .
$$

We substitute this into Eq. (A20) and use Eq. (A16) to remove the $\partial / \partial c\left(\ldots \partial^{2} \xi_{V}(4) / \partial \times \partial u\right)$ term. We also multiply Eq. (A26) by $3 \underset{y}{B} B^{2}$ and integrate with respect to $\zeta$ to obtain an expression for $\oint\left[o\left(\underset{\sim}{J} \cdot \underset{\sim}{B} \underline{B}^{2}\right) a^{2} b_{V}(4) / a x \partial u\right] d \ell / B$. Use of this expression and $E q$. (AzO) leads to

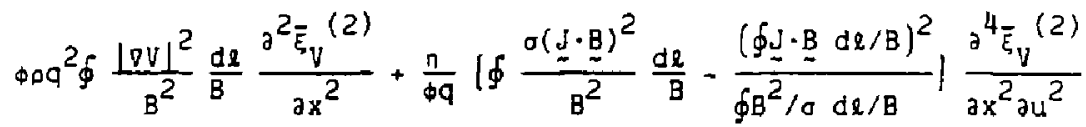

$$
\begin{aligned}
& =\left[\oint \frac{\nabla V \cdot \nabla\left(p_{\perp}+B^{2} / 2\right)}{\tau \sigma B^{2}|\nabla V|^{2}}\left(\left.\sigma \frac{\partial p_{\perp}}{\partial V}\right|_{B}+\left.r \frac{\partial p_{1}}{\partial V}\right|_{B}\right) \frac{d l}{B}+\phi \psi^{\prime} \oint \frac{\sigma J \cdot B}{B^{2}} \frac{\partial}{\partial \zeta} \frac{\nabla V \cdot \nabla u}{|\nabla V|^{2}} \frac{d l}{B}\right.
\end{aligned}
$$

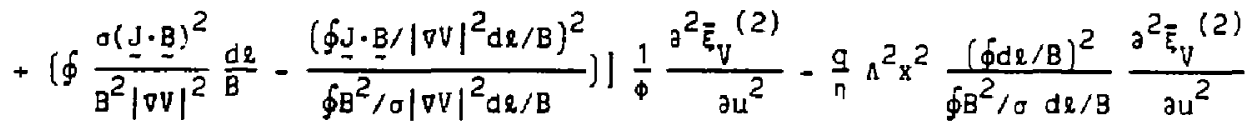

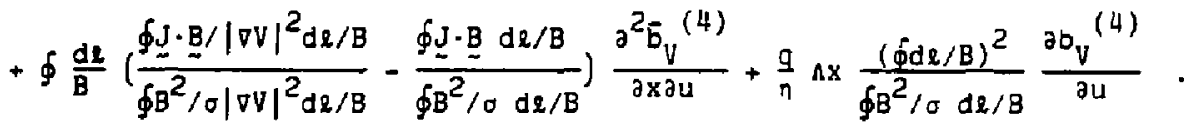

It is useful to use a WKB-type approximation for variations along $u$, mว/วu = ia, define the parameters,

$$
c \equiv\left\langle\frac{B^{2}}{a}\right\rangle\left\langle\frac{B^{2}}{\sigma|\nabla V|^{2}}\right\rangle
$$




$$
\begin{aligned}
& M \equiv \omega^{2}\left\langle\frac{B^{2}}{\sigma|\nabla V|^{2}}\right\rangle\left\langle\frac{|\nabla V|^{2}}{B^{2}}\right\rangle, \\
& K \equiv \frac{\left(\left\langle B^{2} / \sigma|\nabla V|^{2}\right\rangle\right)^{2}}{\Lambda^{2}\left\langle B^{2} / \sigma\right\rangle}\left[\left\langle\frac{\sigma(\underset{\sim}{J} \cdot \vec{\theta})^{2}}{B^{2}}\right\rangle-\frac{(\langle\underline{J} \cdot \underline{B}\rangle)^{2}}{\left\langle B^{2} / \sigma\right\rangle}\right],
\end{aligned}
$$

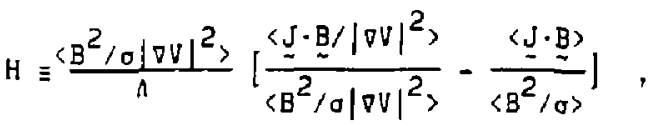

and

$$
\begin{aligned}
& E \equiv \frac{\left.\left.\left\langle B^{2} / \sigma\right| \nabla V\right\rfloor^{2}\right)}{A^{2}:}\left[\left\langle\frac{\nabla V \cdot \nabla\left(p_{\perp}+B^{2} / 2\right)}{\tau \sigma B^{2}|\nabla V|^{2}}\left(\left.\sigma \frac{\partial p_{1}}{\partial V}\right|_{B}+\left.\tau \frac{\partial p_{\|}}{\partial V}\right|_{B}\right)\right\rangle\right. \\
& +\phi \psi^{\prime}\left\langle\left\langle\frac{\sigma J \cdot \underline{B}}{B^{2}} \frac{\partial}{\partial \tau} \frac{\nabla V \cdot \nabla u}{|\nabla V|^{2}}\right\rangle+\left\langle\frac{\sigma(J \cdot B)^{2}}{B^{2}|\nabla V|^{2}}\right\rangle-\frac{\left(\left\langle\underline{J} \cdot \underline{B} /|\nabla V|^{2}\right\rangle\right)^{2}}{\left\langle B^{2} / \sigma|\nabla V|^{2}\right\rangle},\right.
\end{aligned}
$$

with $\langle A\rangle=\oint A d \ell / B / \oint d 2 / B$, introduce the scale lengths,

$$
\begin{aligned}
& x_{0} \equiv\left(\frac{o M n^{2} C^{2}}{\alpha^{2} n^{2}}\right)^{1 / 6}, \\
& Q_{0} \equiv\left(\frac{\alpha^{2} n^{2} \pi C}{o M}\right)^{1 / 3},
\end{aligned}
$$

and set

$$
\begin{aligned}
& x=\gamma X_{0}, \\
& Q=Q Q_{O},
\end{aligned}
$$




$$
\begin{aligned}
& \bar{\xi}_{V}{ }^{(2)}=\Xi, \\
& \bar{b}_{V}{ }^{(4)}=\frac{\text { ian } x_{0}}{\psi^{\prime}} \Psi .
\end{aligned}
$$

Then Eqs. (A28) and (A30) become

$$
\begin{aligned}
& \frac{d^{2} \Psi}{d X^{2}}-H \frac{d E}{d X}=Q(\Psi-X \equiv), \\
& E \equiv+H \frac{d \Psi}{d X}=-\left(Q^{2}-\frac{K}{Q}\right) \frac{d^{2} \equiv}{d X^{2}}-Q X(\Psi-X \Xi) .
\end{aligned}
$$

These are almost the same as Eqs. (40) and (41) of Ref. 4 and Eqs. (9) and (10) of reference $t$ in the limit where the adiabatic constant $y=0$ so that $G$ $=\infty$. The major difference is the introduction of a new term, $(-K / Q) d^{2} \equiv / d x^{2}$, In Eq. (A33). This same modification of the equations would have been obtained in the earlier work with isotropic pressure if the $y=0$ assumption had been made in the beginning. Because of this, $\xi_{B}$ does not enter the equations, except in the inertial term in the E projection of the momentum equation. In the general $Y \neq 0$ case it was convenient to recognize that the divergence of $\xi$ had tc be second order because of the perturbed pressure balance so that $\nabla \cdot \xi^{(2)}$ could be used as an independent variable. We then had to use the parallel component of the momentum equation to eliminate the fourth order perturbed pressure in terms of $\xi_{B}(0)$ and then use information from one order higher in the component of the induction equation. Here we can use the $\nabla V$ component of the induction equation to eliminate $\xi_{V}{ }^{(4)}$ and avoid some of this work. This results in the separation of the parameter $M$ in the earlier work into the two parameters $M$ and $K$ of the present formalism. This 
modification of the equations was found previously in a study of ballooning modes. ${ }^{6}$ The only other difference in these equations from before is the introduction of the parameters $\sigma$ and $\tau$ associated with the pressure anisotropy.

A minor problem should be mentioned. In the $\gamma=0$ anisotropic case, the parallel component of the momentum equation was automatically satisfied because of the conditions imposed by the other equations. Here this coes not seem to occur. It is possible to choose $\xi_{B}$ to eliminate the difficulty, but a better solution should be available.

We can ignore the $K$ term because it is a consequence of the choice of equations of state. Since our purpose is to determine the generalization of $D_{R}$ and not to examine the properties of modes where sound wave propagation can not provide stabilization, we do this and recover the starting point of Glasser's analysis. 1 Then the ideal condition, $D_{I}<0$ for stability, follows from the assumption that $\equiv-X^{S}$ near the rational surface; as $X$ goes to $\infty, s=$ - $1 / 2 \pm(1 / 4-E-H)^{1 / 2}$. The resistive criterion, $D_{R}$ \& 0 for stability, follows from an ordering near marginal stability, $\Psi-1, \equiv-\delta^{-1 / 6}, x-\delta^{1 / 6}$, $Q-\delta^{2 / 3}, E-H-1, D_{R}-\delta$. 


\section{REFERENCES}

${ }^{\prime}$ H.H. Glasser, J.M. Greene, and J.L. Johnson, Phys. Fluids 18, 875 (1976).

${ }^{2}$ M. Kotschenreuther, R.D. Hazeltine, and P.J. Morrison, Phys. Fluids 28, 294 (1985).

3... Connor and R.J. Hastie, Phys. Fluids 19, 1727 (1977).

4J.L. Johnson and J.M. Greene, Plasma Phys. 9, 611 (1967).

${ }^{5}$ C.O. Soies and D.B. Nelson, Phys. Fluids 17, $1879(1974)$.

$6 . \dot{y}$. Connor, R.j. Hastie, T.j. Martin, A. Sykes, and M.F. Turner, in Plasma Fr.ysics and Controlled Nuciear Fusion Research :982 (IAEA, Vierna, 1983). \%oi. III, p. 403.

7B.A. Carreras et al., Phys. Rev. Lett. 50, 503 (1983). 
Or. Frank J. Paoloni, Univ of Wol longong, austral IA

Prot, M.H. Grennan, Univ Syanoy, AustRalia

Plasma Research Lab., Australi an Nat. Univ., austral IA

Prot, I,R, Jones, Flinders Univ., AuSTRALIA

Prof. F. Cao, Ingt Theo Phys, AustRia

Prof. M. Heindler, Institut fur Theoretisehe Physik, AUSTRIA

M. Goossens, Astronomisen Instituut, BELGIUM

Ecole Royale Miliraire, iab de Phys Plasmas, BELGIuy

Commission-Eurogean, Og-X 11 fusion Prog, BELGIUM

Prof. R. Boucique, Laboratorium voor Natuurkunde, BELGIUM

Or. P.H. Sakanaka, Instituto Fisica, BRAzIL

Instituto de Pesquisas Espaciasi-INPE, BRAzIL

Documents Office, Atomic Energy of Canada Libitad, CanaDa

Or. M.P. Bactiynski, Mpg Technologies, Inc., CAMAOA

Or. H.M. Skarsgard, University of Saskatchewar, CANAOA

Cr. H. Barnard, University of British Columbia, CANMOA

Prof. J. Teichmann, univ. of Montradi, CakaOa

Prof. S.R. Sreenivasan, University of Calgary, CANADA

Prof. Tucor W. Johnston, INRS-Energie, CaNADA

Dr. C.R. James, Univ, ot Alberta, CAMAOA

Dr. Peter Lukac, Komenskeho Univ, CZEChosLOVAKIA

The Librarian, Culhan Laboratery, ENGLANO

The Librarian, Rurhertord Appleton Laboratory, ENGLAND

Mrs. S.A. Huteninson, JET Library, ENGLANO

C. Mouttet, Lab. de Physique des Mllieux Ionises, France

J. Radat, CEN/CADARACHE - Bat 506, fRAMCE

Univ. of loannina, Library of Physics Dept. GREECE

Dr. Ton Mual, Academy Bibliographic Ser., HONG KONG

Preprint Library. Hungarian Acadeny of Sciences, HUMGary

Dr. B. Dasgupta, Sana Inst of Nucl, Phys.. INDIA

Dr. P. Kaw, Instifuto for Plasina Researen, INDIA

Dr. Philip Rosenau, Israel Inst. Tech, ISRAEL

Librarian, Int'I Ctr theo Phys, ITALY

Prot. G. Rostagni, Univ Oi Padova, ITALY

Miss Clelia de Palo, assoc EURATOH-ENEA, ITALY

Bibliateca, instituta di fisica del Plasma, ITALY

Dr. H. Yamoto, Toshida Res \& Dav, JAPAN

Prot. 1. Kawakani, Atomic Energy Res. Instituto, JaPAN

Prot. Kyoji Nishikawa, Uniy of Hiroshima, JAPAN

Direc. Dept. Large Toksmak Res. JAERI, JAFAN

Prot, Satoshi Iton, Kyushu University, JAPAM

Research !nfo Canter, Nagoya University, JAPAN

Prof. S, Tanako, Kyoto Univergity, JAPAN

Library, Kyoto University, JAPAN

Prof. NODUyuki inout, University of TOKYO, JAPAN

5. Wori, JAERI, JAPAN

Librarian, Kores Advanced Enargy Ras. Institute, KOREA

Prot. 0.1. Choi. Adr. Inst Sei \& Teeh, KOREA

Prot, 9.S. Liloy. University of Waikato, NEW ZEALANO

Institute of Plasad Physics, PEOPL's REPJBLIC OF OMINA

Librarian, institute of Phys., PEOPLE'S REPUBLIC OF CHINA

Library, Tsing Hua University, PEOPLE'S REPUBL IC OF CHINA
Z. Li, SOuthwest Inst. Physics, PEOPLE'S REPUBLIC OF CHINA Prof. J.A.C. Cabral, Inst Suderior Tecnico, PORTugal Dr. Oetavisn Potrus, AL I CUzA University, ROMANia Dr. Johan de Villiers, Fusion Studies, AEC. SO AFRICA Prof. M.A. Hellberg, University of Naral, SO AFRICA C.I.E.M.A.T., Fusion Div. Library, SPAIN Dr. Lennart Stenflo, University of LMEA, SWEDEN Library, Royal Inst Tech, SWEDEN

Prof. Hans Wilnglmson, Chalmers Un iv Tech, SWEDEN

Cantro Phys des Plasmas, Ecolo Polytech Fed, SwITZERLANo Bibliotheek, Fom-Inst Voor Plasma-Fysica, THE NETHERLANDS Dr. D.D. Ryutov, Siberian Acad Sci, USSR

Dr. G.A. Eliseev, Kurchatov Institute, USSR

Or. Y.A. Glukhikh, Inst Electrophysical Appararus, USSR

Dr. Y.T. Tolok, Inst. Phys, Tech. USSR

Or. L.M. Kovrizhnykh, Institute Gen. Physics, USSA

Nuclear Ros. Estadismment, Julich Lta., W. GERMANY

Bibliornek, Inst. Fur Plasmafarschung, W. GERMANY

Or. K. Sehindler, Ruhr Un iversitar Buehum, id. GERMANY

ASDEX Reading Rom, I Do.sya-rianck-Instiqut fur

PIasmadhysik, W. GERMANY

Librarian, Max-P! anek Institut, M. GERMANY

Prot. R.K. Janer, inst Phys, YugOSLAvIA 